\title{
The roseoloviruses downregulate the protein tyrosine phosphatase PTPRC (CD45)
}

\author{
Melissa L. Whyte ${ }^{1}$, Kelsey Smith ${ }^{1}$, Amanda Buchberger ${ }^{2,4}$, Linda Berg Luecke ${ }^{4}$, Lidya \\ Handayani Tjan ${ }^{3}$, Yasuko Mori ${ }^{3}$, Rebekah L Gundry ${ }^{2,4}$, and Amy W. Hudson ${ }^{1 \#}$
}

\begin{abstract}
1: Department of Microbiology and Immunology, Medical College of Wisconsin, Milwaukee, WI 2. Department of Biochemistry, Medical College of Wisconsin, Milwaukee, WI

3. Division of Clinical Virology, Kobe University Graduate School of Medicine, Kobe, Japan 4: Current address: CardiOmics Program, Center for Heart and Vascular Research; Division of Cardiovascular Medicine; and Department of Cellular and Integrative Physiology, University of Nebraska Medical Center, Omaha, NE
\end{abstract}

\#To whom correspondence should be addressed: ahudson@mcw.edu

Runnning title: Roseolovirus downregulation of CD45 


\section{Abstract}

48 Like all herpesviruses, the roseoloviruses (HHV6A, -6B, and -7) establish lifelong

49 infection within their host, requiring these viruses to evade host anti-viral responses.

50 One common host-evasion strategy is the downregulation of host-encoded, surface-

51 expressed glycoproteins. Roseoloviruses have been shown to evade host the host

52 immune response by downregulating NK-activating ligands, MHC class I, and the

53 TCR/CD3 complex. To more globally identify glycoproteins that are differentially

54 expressed on the surface of HHV6A-infected cells, we performed cell surface capture of

$55 \mathrm{~N}$-linked glycoproteins present on the surface of T cells infected with HHV6A, and

56 compared these to proteins present on the surface of uninfected T cells. We found that

57 the protein tyrosine phosphatase CD45 is downregulated in T cells infected with

58 HHV6A. We also demonstrated that CD45 is similarly downregulated in cells infected

59 with HHV-7. CD45 is essential for signaling through the T cell receptor and as such, is

60 necessary for developing a fully functional immune response. Interestingly, the closely

61 related $\beta$-herpesviruses human cytomegalovirus (HCMV) and murine cytomegalovirus

62 (MCMV) have also separately evolved unique mechanisms to target CD45. While

63 HCMV and MCMV target CD45 signaling and trafficking, HHV6A acts to downregulate

64 CD45 transcripts.

\section{Importance}

66 Human herpesviruses- 6 and -7 infect essentially $100 \%$ of the world's population before

67 the age of 5 and then remain latent or persistent in their host throughout life. As such, 68 these viruses are among the most pervasive and stealthy of all viruses. Host immune 
69 cells rely on the presence of surface-expressed proteins to identify and target virus-

70 infected cells. Here, we investigated the changes that occur to proteins expressed on

71 the cell surface of T cells after infection with human herpesvirus-6A. We discovered

72 that HHV-6A infection results in a reduction of CD45 on the surface of infected cells.

73 Targeting of CD45 may prevent activation of these virus-infected T cells, possibly

74 lengthening the life of the infected T cell so that it can harbor latent virus. 


\section{Introduction}

76 Human Herpesvirus-6A (HHV6A) is a human-specific, T cell-tropic $\beta$-herpesvirus that is

77 most closely related to the other members of the roseolovirus genus, HHV-6B and HHV-

78 7, as well as human cytomegalovirus (HCMV). Primary infections with HHV-6 and -7

79 usually occur before the age of three and are often characterized by a high fever $(1,2)$.

80 HHV-6 and -7 infect over $90 \%$ of the population, and like other herpesviruses, HHV-6

81 and -7 remain latent or establish lifelong infections in their hosts. As such, these viruses

82 are among the most pervasive and stealthy of all viruses; they must necessarily excel at

83 escaping immune detection throughout the life of the host, yet little is known about how

84 these viruses so successfully escape host defenses.

85 The ability of host immune cells to detect virus-infected cells is largely dependent on

86 interactions between proteins expressed on the surface of immune cells and those

87 expressed on the surface of their targets. Viruses have necessarily evolved to alter the

88 expression of these cell-surface-expressed proteins to evade detection by the host

89 immune system. The herpesviruses, particularly the cytomegaloviruses, have long been

90 known to employ devious strategies to interfere with expression of host-encoded

91 surface-expressed proteins. For example, most herpesviruses, including HHV-6 and -7,

92 interfere with antigen presentation by downregulating class I major histocompatibility

93 complex $(\mathrm{MHC})$ molecules from the surface of infected cells (4-13). Additionally, HCMV,

94 the most closely-related virus to the roseoloviruses, encodes at least 5 different gene

95 products and a miRNA that all participate in downregulation of natural killer (NK)-

96 activating ligands, all in an effort to prevent natural killer cells from identifying and killing

97 infected cells (14-18). We have found that HHV6A, -6B, and -7 each encode a single 
98 gene product, U21, that acts as a multifunctional transmembrane glycoprotein to

99 downregulate not only multiple NK activating ligands, but also most class I MHC alleles

$100 \quad(12,19-21)$

101 Herpesviruses also target surface-expressed proteins to hinder the ability of immune

102 cells to perform their effector function. For example, herpesviruses inhibit T cell function

103 through the downregulation of co-stimulatory ligands like B7 and CD40, and

104 upregulation of co-inhibitory ligands such as PD-L1 and galectin-9 (22-32).

105 Herpesviruses also downregulate adhesion molecules, such as I-CAM, V-CAM, PECAM

106 and ALCAM, which can physically disrupt formation of the immune synapse and inhibit

107 T cell activation (33-37).

108 Since most herpesviruses are not T cell-tropic, changes in surface expression occur in 109 infected cells, and infected cells then exert their immunosuppressive effects through

110 interaction with uninfected T cells. But what happens to the surface of a T cell when it is

111 infected with a herpesvirus? The T cell-tropic roseoloviruses allow us to explore and

112 learn from alterations to the glycoprotein landscape on the surface of herpesvirus-

113 infected T cells. Herein, we applied a mass spectrometry strategy to identify and

114 quantify $\mathrm{N}$-linked glycoproteins on the cell surface of HHV6A-infected T cells to yield the

115 first insights into how virus infection induces alterations in the cell surface landscape of

116 host cells. We discovered that the protein tyrosine phosphatase CD45 (PTPRC) is

117 downregulated from the surface of HHV6A-infected T cells. CD45 is expressed on the

118 surface of all nucleated cells of hematopoietic origin, where its activity is critical for the

119 proper function of immune cells (reviewed in (39-42)). The phosphatase activity of CD45

120 is required for successful signaling through the TCR (43-45), and as such, the 
121 downregulation of CD45 could be an attractive strategy for viruses to impair T cell

122 signaling.

\section{Results}

\section{Cell-surface capture of CD45 in HHV6A-infected T cells}

125 To identify host-encoded surface-expressed proteins targeted by HHV6A, we performed

126 cell surface capture (CSC), a chemoproteomic approach for the specific identification of

127 cell surface $N$-glycoproteins (46-50). Using CSC and bioinformatic analysis tools, we

128 compared N-linked glycoproteins identified on the surface of T cells infected with

129 HHV6A to those identified on the surface of uninfected T cells $(3,38)$. To minimize

130 biological variability, we performed CSC in HHV6A-infected cell line. HHV6A infects

131 only a small number of cultured cell lines, and for these studies, we used JJhan cells, a

132 CD4+ T cell line variant of Jurkat cells (51).

133 We identified 605 cell surface $\mathrm{N}$-linked glycoproteins on the surface of infected and

134 uninfected JJhan cells, including 594 human and 11 viral proteins. Strikingly, we

135 identified 47 unique peptides derived from the protein tyrosine phosphatase PTPRC

136 (CD45), spanning all $22 \mathrm{~N}$-linked glycosylation sites (Fig. 1a). CD45-derived peptides

137 were consistently less abundant on the surface of HHV6A-infected JJhan cells across

138 multiple biological and technical replicates (Fig. 1b-d), suggesting that CD45 is

139 downregulated from the surface of HHV6A-infected JJhan cells. 
141 To independently validate the

142 downregulation of CD45

143 observed in our mass

144 spectrometry experiments, we

145 examined surface-expression of

146 CD45 in HHV6A-infected cells by

147 flow cytometry. We infected

148 JJhan cells with a recombinant,

149 bacterial artificial chromosome

150 (BAC)-derived HHV6A virus

151 encoding soluble green

152 fluorescent protein (GFP), which

153 allowed us to identify actively-

154 infected cells (52). In uninfected

155 JJhan cells, we observed a single

156 population of CD45-expressing

157 cells, while in HHV6A-infected

158 JJhan cells, we observed a

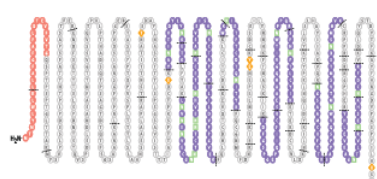

Extracellular
( $\mathrm{N}$-glycopeptide identified

$N$-glcosylation site identified

Known sequence variants

Signal peptide

Trypsin cleavage sites
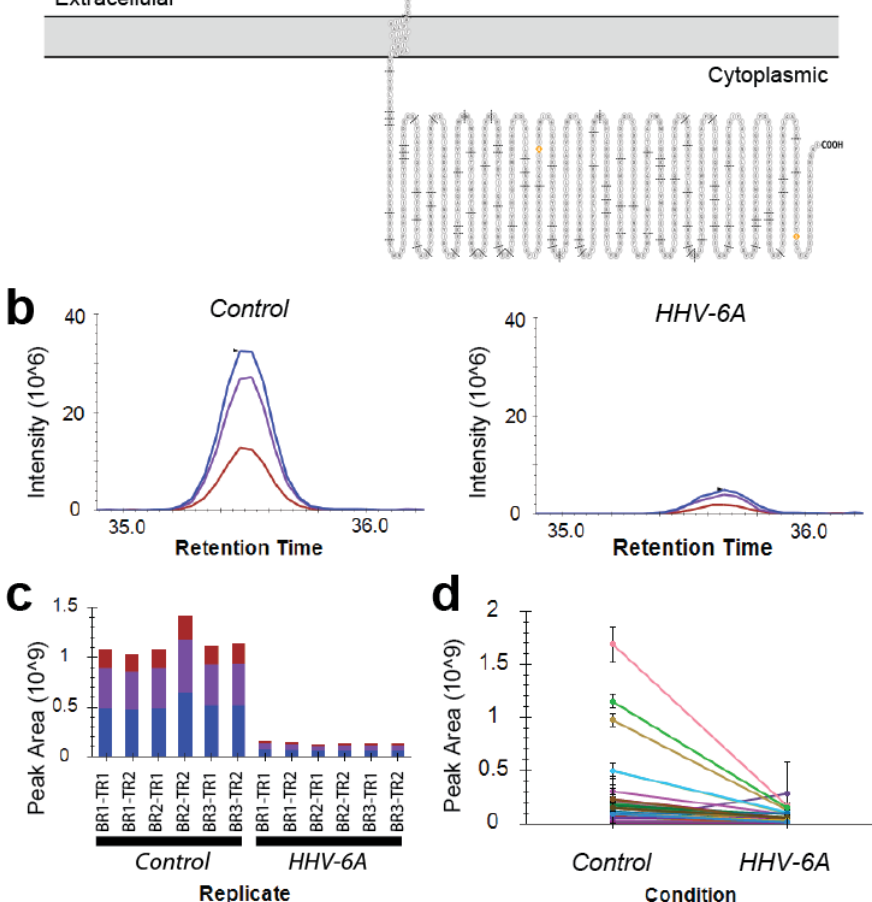

Figure 1. Mass spectrometry data for CD45. (a) Graphical representation of CD45 protein with extracellular N-linked glycopeptides identified by CSC indicated. This approach specifically enriches and identifies $\mathrm{N}$-glycopeptides from the extracellular domain of cell surface proteins. Image generated using Protter (3). (bd) Label-free quantitation data for CD45. Representative peaks of a peptide (YAnITVDYLYNK, where $\mathrm{n}$ is the site of $\mathrm{N}$-glycosylation) detected across individual replicate analyses (b) and three replicate analyses (including technical replicates) (c) for each sample group, showing CD45 is more abundant in control compared to infected cells. Each color (blue, purple, and red) are associated with the peak area from the $[M]+0,[M]+1$, and $[M]+2$ isotopic peaks, respectively. $[M]$ is the monoisotopic mass. (d) Summary of peak areas for all 67 peptide observations for CD45 across all technical and biological replicates. Each line is a different peptide. BR: biological replicate; TR: technical replicate. Results shown in b-d were generated using Skyline (38).

159 reduced surface expression of CD45 (Fig. 2a), consistent with our mass spectrometry

160 finding that the presence of CD45 is diminished on the surface of HHV6A-infected cells.

161 CD45 is expressed as multiple different isoforms in human cells (53-55). To determine

162 whether all isoforms of CD45 are downregulated in HHV6A-infected JJhan cells, we

163 labeled cells with antibodies directed against the CD45 isoforms commonly expressed 

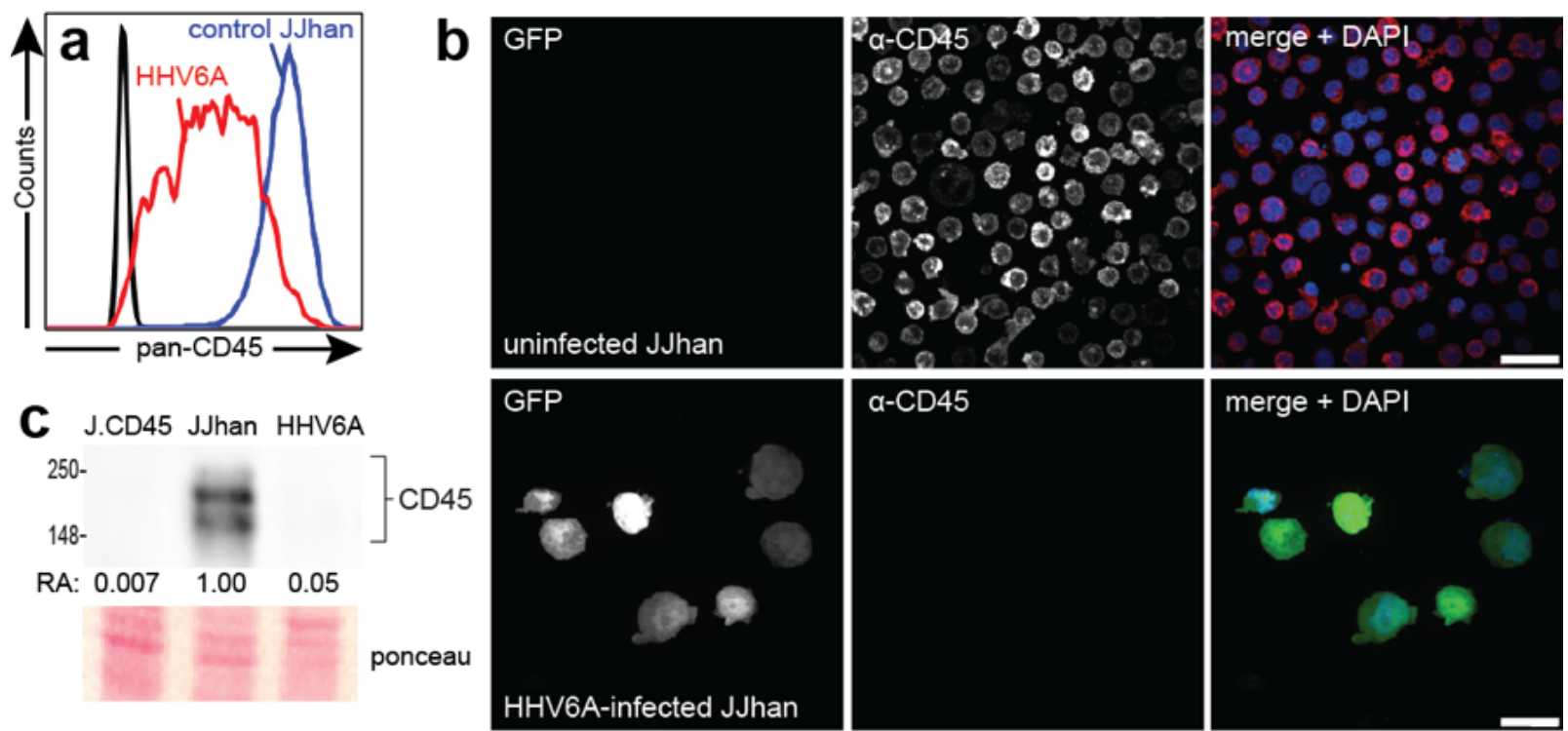

Figure 2. CD45 is downregulated in HHV6A-infected cells. (a) Flow cytometric analysis of HHV6A-infected JJhan cells (red) and uninfected JJhan cells (blue). HHV6A-infected cells were gated on as GFP+. Live cells were labeled with an antibody directed against CD45. (b) Confocal immunofluorescence microscopy images showing uninfected JJhan cells (top panels) and HHV6A-infected (GFP+) JJhan cells (bottom panels) that were fixed, permeabilized, and labeled with an antibody directed against CD45. Images were taken at 100X magnification and are shown as maximum intensity projections. Scale bar $=20 \mu \mathrm{m}$. (c) Immunoblot analysis of CD45 in whole cell lysates generated from J.CD45 cells (negative control), uninfected JJhan cells, and HHV6A-infected JJhan cells. Protein was normalized to total protein for each lane. RA : relative abundance. Abundance is calculated relative to uninfected JJhan cells. Ponceau shows total protein loaded per lane.

164 in Jurkat cells, the parental cell line of JJhan cells (56). CD45-RO, -RB, and -RC were

165 all downregulated in HHV6A-infected JJhan cells relative to uninfected JJhan cells (Fig.

166 S1), suggesting the downregulation of CD45 from the surface HHV6A-infected JJhan

167 cells is not specific to any one CD45 isoform.

168 Viruses often reroute the intracellular trafficking of surface-expressed proteins from the

169 cell surface to alter host cell biology. For example, the HHV6A U24 gene product

170 downregulates the T-cell receptor (TCR)/CD3 by stimulating endocytosis of the receptor

171 resulting in relocalization of TCR/CD3 from the cell surface to endosomes, and U21

172 reroutes class I MHC molecules to lysosomes $(13,57)$. We therefore sought to

173 determine whether the reduction in surface-expressed CD45 observed in HHV6A-

174 infected cells was the result of a redistribution of CD45 within the cell. To examine the

175 localization of CD45, we performed immunofluorescence microscopy of permeabilized 
176 cells labeled with an antibody directed against human CD45. As expected, CD45 was

177 localized to the plasma membrane in uninfected JJhan T cells. In HHV6A-infected

178 JJhan cells, however, we observed a striking disappearance of CD45 labeling (Fig. 2b).

179 To better quantify the downreguation of CD45 in HHV6A-infected JJhan cells, we next

180 examined steady-state levels of CD45 protein by immunoblot analysis of whole-cell

181 lysates generated from uninfected or HHV6A-infected JJhan cells. Consistent with our

182 immunofluorescence microscopy data (Fig. 2b), immunoblot analysis showed a 95\%

183 reduction in CD45 protein in HHV6A-infected JJhan cells relative to uninfected JJhan

184 cells (Fig. 2c). Taken together, these results demonstrate that CD45 is not only

185 downregulated from the surface of HHV6A-infected JJhan cells but is also depleted

186 from HHV6A-infected cells.

187 CD45 expression in HHV7-infected T cells

188 The HHV6A genome is almost entirely co-linear with the two other roseolovirus

189 genomes HHV-6B and HHV-7 $(58,59)$. As such, we reasoned that HHV-6B and HHV-7

190 infection may also result in CD45 downregulation. To determine whether CD45 is

191 downregulated in HHV7-infected cells, we evaluated the localization of CD45 in HHV7-

192 infected SupT1 cells by immunofluorescence microscopy. Since a recombinant GFP-

193 containing BAC containing the coding sequence of $\mathrm{HHV}-7$ is not yet available, we

194 identified HHV7-infected cells using an antibody directed against the U21 gene product

195 from HHV-7 (Fig. 3, green). Similar to cells infected with HHV6A (Fig. 2b), HHV7-

196 infected SupT1 cells showed reduced CD45 labeling (Fig. 3, bottom panels as 
197 compared to

198 uninfected

199 SupT1 cells

$200 \quad$ (Fig. 3, top

201 panels),

202 suggesting that

203 the ability to

204 downregulate

205 CD45 is

206 common to all

207 roseoloviruses.
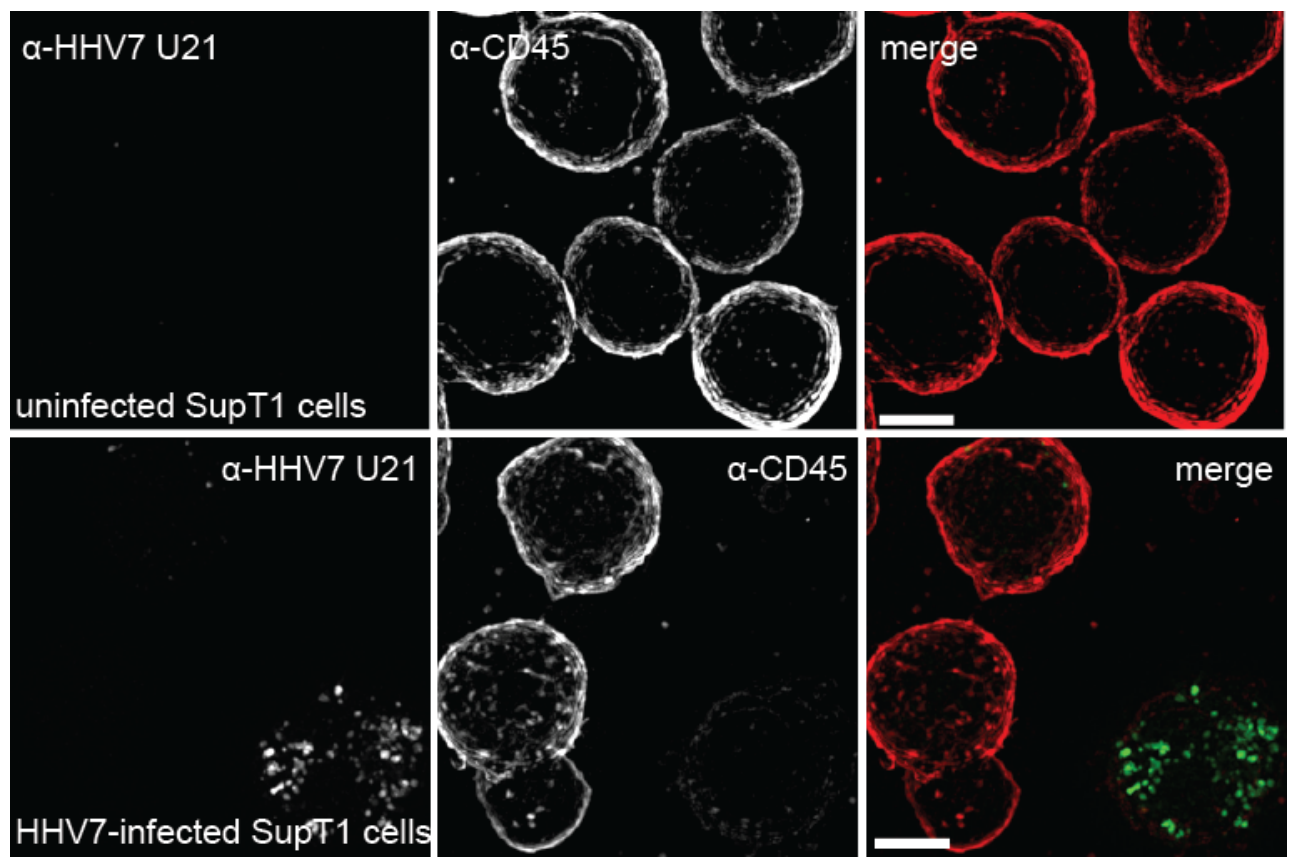

Figure 3. CD45 is downregulated in HHV7-infected T cells. Structured Illumination 3-dimensional microscopy images showing uninfected control T cells (top panels) and HHV7-infected T cell (bottom panels). Fixed and permeabilized cells were labeled with an antibody directed against the U21 open reading frame (ORF) from HHV7 (green) and an antibody directed against human CD45 (red). Images were taken at 100X and are shown as maximum intensity projections. Scale bar $=5 \mu \mathrm{m}$.

209 Viruses often employ the strategy of degrading host proteins involved in the host

210 response to virus infection. For example, the murine cytomegalovirus m42 gene product

211 induces internalization and subsequent proteasomal degradation of CD45 (60). To

212 further investigate the mechanism by which CD45 is downregulated in HHV6A-infected

213 JJhan cells, we examined the stability of CD45 in the presence of proteasomal and

214 lysosomal protease inhibitors.

215 Lysosomal protease function is compromised at a more neutral $\mathrm{pH}$, thus stabilization of

216 a protein in the presence of the weak base ammonium chloride $\left(\mathrm{NH}_{4} \mathrm{Cl}\right)$ would suggest

217 the involvement of lysosomal proteases in its turnover. Likewise, stabilization of a

218 protein in the presence of the peptide-aldehyde MG-132, which selectively inhibits 
219 proteolytic activity of the $26 \mathrm{~S}$

220 proteasome, would suggest

221 involvement of proteasomal

222 proteases in the turnover of that

223 protein. We separately inhibited

224 lysosomal and proteasomal

225 protein degradation, treating

226 cells with $\mathrm{NH}_{4} \mathrm{Cl}$ or $\mathrm{MG}-132$, and

227 assessed steady-state CD45

228 protein levels by immunoblot

229 analysis. In cells treated with a

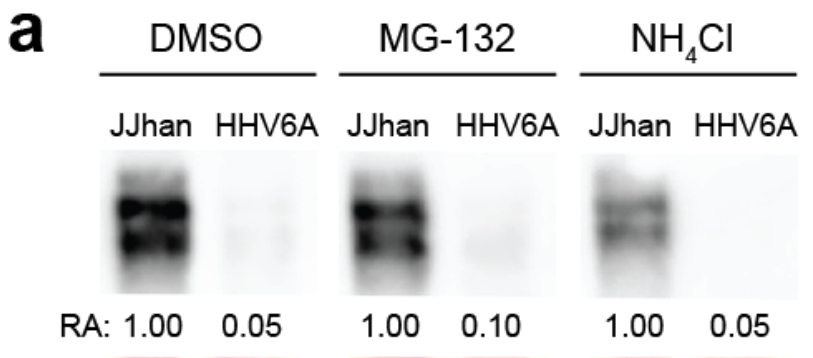

Ponceau

230 DMSO vehicle control, we

231 observed a $95 \%$ reduction in

232 CD45 abundance in HHV6A-

233 infected cells (Fig 4a), which is

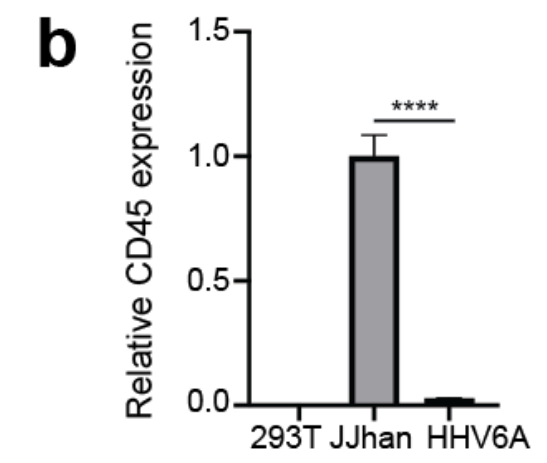

Figure 4. CD45 is downregulated at the RNA level in HHV6A-infected T cells. (a) Immunoblot analysis showing steady-state abundance of CD45 protein in uninfected JJhan cells, or HHV6A-infected cells under different conditions (DMSO vehicle control, MG-132, or $\mathrm{NH} 4 \mathrm{Cl}$ treatment for 19 hours). Equal protein was loaded in each lane, and CD45 abundance was calculated relative to uninfected JJhan cells for each treatment. RA = relative abundance. Ponceau-stained lanes visually illustrate equal protein loading. (b) CD45 RNA abundance in 293T cells (negative control), uninfected JJhan cells, or HHV6A-infected cells. CD45 RNA abundance is calculated relative to a cellular control gene and shown here relative to uninfected JJhan cells. Data shown is from a single representative biological replicate, performed in technical triplicate for each sample, mean $+/-\mathrm{SD}$. ${ }^{* \star *}=p$ $<0.0001$.

234 similar to what we observed in untreated cells (Fig. 2c), suggesting the dimethyl

235 sulfoxide (DMSO) vehicle does not affect degradation of CD45. We observed a similar

236 reduction in CD45 in cells treated with MG-132 or $\mathrm{NH}_{4} \mathrm{Cl}$ (Fig. 4a).

237 We also examined localization of CD45 in HHV6A-infected cells treated with MG-132 or

$238 \mathrm{NH}_{4} \mathrm{Cl}$ by immunofluorescence microscopy. As expected, we observed surface-

239 localization of CD45 in untreated, uninfected JJhan cells (Fig. S2, panel a). We also

240 observed surface-localization of CD45 in uninfected JJhan cells treated with a DMSO

241 vehicle, MG-132, or $\mathrm{NH}_{4} \mathrm{Cl}$ (Fig. S2, panels b, c, and d, respectively), suggesting CD45 
242 localization is not affected by treatment with MG-132 or $\mathrm{NH} 4 \mathrm{Cl}$ outside the context of an

243 HHV6A infection. Consistent with the immunoblot data, there was little to no CD45

244 labeling present in untreated HHV6A-infected JJhan cells (Fig. S2, panel e). Similarly,

245 there was little to no CD45 labeling in HHV6A-infected treated with a DMSO vehicle,

246 MG-132, or $\mathrm{NH} 4 \mathrm{Cl}$ (Fig. S2, panels f, g, \& h, respectively). Taken together, these results

247 suggest that the downregulation of CD45 in HHV6A-infected JJhan cells occurs by

248 some method other than protein degradation.

249 CD45 gene expression in HHV6A-infected T cells

250 Because CD45 was not stabilized in the presence of proteasomal or lysosomal

251 inhibitors, we hypothesized that CD45 was downregulated at the transcript level in

252 HHV6A-infected T cells. To test this hypothesis, we quantified CD45 mRNA in

253 uninfected and HHV6A-infected JJhan cells by quantitative reverse transcriptase-

254 polymerase chain reaction (qRT-PCR). CD45 mRNA levels were greatly reduced in

255 JJhan cells infected with HHV6A as compared to uninfected JJhan cells (Fig. 4b),

256 suggesting that CD45 transcripts are downregulated in HHV6A-infected JJhan cells.

\section{Discussion}

258 Herpesviruses, as life-long pathogens, are especially masterful at reprogramming host

259 cells to create a more hospitable environment. Perhaps the greatest challenge to a virus

260 is the detection and killing of its host cell by immune cells. As discussed, the

261 herpesviruses encode multiple gene products that alter host cell biology to prevent the

262 identification of infected cells. These measures are not entirely sufficient, however, and

263 viruses also strategize to target immune cells to inhibit their functional capacity. One 
264 way viruses can inhibit these processes is by targeting the proteins that are important

265 for immune cell function, such as the protein tyrosine phosphatase CD45.

266 CD45 is expressed on the surface of all nucleated cells of hematopoietic origin, where

267 its activity is critical for the proper function of immune cells (reviewed in (39-42)). In T

268 cells, CD45's primary substrates are Src family kinases (61-63). CD45

269 dephosphorylates the inhibitory phosphotyrosine residue on the Src kinase Lck, leaving

270 the kinase in a 'primed' state, so that it can be activated through T cell receptor (TCR)

271 signaling (62). The phosphatase activity of CD45 is required for successful signaling

272 through the TCR (43-45), and as such, the downregulation of CD45 could be an

273 attractive strategy for viruses to inhibit T cell signaling.

274 Here we describe the downregulation of CD45 by two roseoloviruses, HHV6A and HHV-

275 7. Expression of CD45 is markedly reduced in HHV6A-infected JJhan cells and HHV7-

276 infected SupT1 cells. While we do not yet fully understand the functional consequences

277 of CD45 downregulation in roseolovirus-infected cells, we can gather clues from three

278 other viruses also known to target CD45: human cytomegalovirus (HCMV), human

279 adenovirus, and murine cytomegalovirus (MCMV).

280 CD45 is targeted by HCMV pUL11 (64). The extracellular domain of pUL11, which is

281 expressed on the surface of HCMV-infected epithelial cells or fibroblasts, interacts with

282 CD45 on nearby uninfected T cells. The interaction between pUL11 and CD45 inhibits

283 the phosphatase activity of CD45, which in turn impairs TCR signaling and ultimately, T

284 cell proliferation $(64,65)$. Inhibition of CD45 by pUL11 also results in an increase in

285 production of the anti-inflammatory cytokine IL-10 (65). In HHV6A infection, the 
286 concentration of secreted IL-10 protein was shown to be increased during HHV6A

287 infection at timepoints up to 72 hours post-infection $(66,67)$. Since the inhibition of

288 CD45 during HCMV infection results in an increase in IL-10 production (65), it is

289 tempting to speculate that the downregulation of CD45 we observe in HHV6A-infected T

290 cells may be involved in the increase in IL-10 levels shown to occur during HHV6A

291 infection.

292 Another virus that devotes an open reading frame to the downregulation of CD45 is

293 Adenovirus19a (Ad19a). Ad19a E3/49K protein is cleaved to a secreted form (sec49K)

294 that interacts with CD45 on nearby uninfected NK cells and T cells. Sec49K-mediated

295 inhibition of CD45 suppresses T cell activation and signaling, resulting in diminished

296 production of the anti-viral cytokine IFN-y, and inhibition of NK cell activation (68). After

297 HHV6A infection of stimulated peripheral blood mononuclear cells, the production of

298 IFN- $y$ was also reported to be reduced (66). Since inhibition of CD45 function during

299 adenovirus infection results in a decrease in IFN-Y production (68), it is possible that the

300 decrease reported in IFN-y production during HHV6A infection may occur as a result of

301 CD45 downregulation in infected T cells.

302 Unlike HCMV pUL11 and Ad19a sec49K, which act extracellularly to inhibit CD45,

303 MCMV encodes a protein, m42, that induces the internalization and degradation of

304 CD45 within MCMV-infected macrophages (60). MCMV m42 acts as an adaptor or

305 activator of HECT3 E3 ubiquitin ligases, and through ubiquitination, m42 marks CD45

306 for lysosomal degradation (60). The functional outcome of m42-mediated

307 downregulation of CD45 in MCMV-infected macrophages is unclear. HHV6A-mediated

308 downregulation of CD45 is similar to MCMV-mediated downregulation of CD45, in that 
309 these viruses downregulate CD45 from within infected cells, as opposed to acting on

310 CD45 in trans on the surface of nearby uninfected cells. As yet, the functional outcome

311 of downregulating CD45 within either of these infected immune cells remains elusive.

312 The roseolovirus genomes lack positional or functional homologs of MCMV m42 or

313 HCMV pUL11, and unlike HCMV, MCMV, and Ad19a, HHV6A infection results in the

314 dramatic transcriptional downregulation of CD45. HHV6A therefore downregulates

315 CD45 by a novel mechanism. The separate evolution of four unique mechanisms to

316 target a single host protein strongly suggests that CD45 is an important viral target,

317 though its impact is unclear: how might the roseoloviruses benefit from the

318 downregulation of CD45 in infected T cells? As discussed above, while the influence of

319 CD45 downregulation on cytokine production would certainly benefit HHV6A, it is

320 important to note that HHV6A preferentially infects T cells. Since it takes days to mount

321 a virus-specific T cell response, during a primary infection, the T cells that HHV6A

322 infects are not likely to be HHV6A-specific. Therefore, downregulation of CD45 in

323 infected T cells would not directly impair activation of T cells responding to HHV6A

324 infection. Instead, downregulation of CD45 may be a means to inhibit activation of the

325 HHV6A-infected T cell, possibly preventing activation-induced cell death, and creating a

326 host cell environment conducive to harboring latent virus. Future work is focused on

327 identification of the HHV6A gene products involved in the transcriptional regulation of

328 CD45 and exploring the functional consequences of CD45 downregulation in

329 roseolovirus-infected T cells. 


\section{Materials \& Methods}

\section{Cell lines and viruses}

333 JJhan, ND10 depl JJhan, and J.CD45 T cells were cultured in RPMI-1640 medium

334 (ThermoFisher Scientific, Waltham, MA) supplemented with $5 \%$ fetal bovine serum

335 (FBS) and 2 mM L-glutamine. JJhan cells depleted of ND10 (ND10 depl JJhan cells) were

336 the kind gift of Dr. Benedikt Kaufer (Freie Universität, Berlin, Germany) (69). J.CD45

337 cells (CD45 -negative Jurkat cells) were kindly provided by Dr. Arthur Weiss (UCSF,

338 San Francisco, CA) (70). 293T cells were cultured in Dulbecco's modified Eagle

339 medium (DMEM) (Invitrogen, Carlsbad, CA) supplemented with 5\% FBS, 5\% newborn

340 calf serum, and $2 \mathrm{mM}$ L-glutamine. The HHV6A virus used in these studies is a

341 recombinant HHV6A virus (strain U1102) generated from a bacterial artificial

342 chromosome (BAC) containing the HHV6A genome with GFP inserted between the U53

343 and U54 ORFs (71). Infectious HHV6A virus was generated by electroporating HHV6A-

344 GFP BAC DNA into JJhan T cells. HHV6A virus was propagated by mixing uninfected

345 ND10-depleted Jhan cells with infected JJhan cells once HHV6A-infected JJhan cells

346 were $>80 \%$ GFP+. HHV6A-infected and uninfected JJhan cells as well as J.CD45 cells

347 were stimulated with $3.75 \mathrm{ug} / \mathrm{ml}$ Phytohemagglutinin (PHA-P) and $9 \mathrm{ug} / \mathrm{ml}$

348 hydrocortisone. HHV6A-infected cells used for experiments in this study were $\geq 70 \%$

349 GFP+ at the time of harvest. SupT1 cells were cultured in RPMI-1640 medium

350 supplemented with $2.5 \%$ FBS and $2 \mathrm{mM} \mathrm{L-Glutamine.} \mathrm{HHV-7} \mathrm{infection} \mathrm{(strain} \mathrm{SB)} \mathrm{was}$

351 performed by co-culture of HHV7-infected SupT1 cells with uninfected SupT1 cells. 
354 The monoclonal anti-human CD45 antibody (clone S5-Z6, Santa Cruz Biotechnology,

355 Dallas, TX) was used for flow cytometry $\left(1 \mathrm{ug} / 1 \times 10^{6}\right.$ cells $)$, immunofluorescence

356 microscopy (1:50), and immunoblotting (1:50). The monoclonal anti-CD45 antibody

357 (clone MEM-28, Millipore-Sigma, St. Louis, MO) was used for immunofluorescence

358 microscopy (1:200). The polyclonal antibody MCW62 (U21-N) was raised against the N-

359 terminus of HHV-7 U21 (1:400) (72). AlexaFluor-405, -488, -594, and -647-conjugated

360 goat-anti mouse and rabbit secondary antibodies were used at dilutions recommended

361 by the manufacturer (ThermoFisher Scientific). Chemicals were purchased from Sigma

362 Millipore unless otherwise noted.

363 Identification of cell surface N-glycoproteins

364 Cell Surface Capture (CSC) was applied to control JJhan and HHV6A-infected JJhan T

365 cells (10 million cells per replicate, three biological replicates per condition). Briefly,

366 extracellular glycans on intact cells were oxidized using sodium meta-periodate, and the

367 resulting aldehydes were labelled with biocytin hydrazide to form a 'handle' for

368 enrichment. Cells were then lysed, proteins enzymatically digested, and biotinylated

369 glycopeptides were enriched using streptavidin beads. N-glycopeptides were then

370 selectively released by Peptide-N-Glycosidase F (PNGase:F) and analysed by mass

371 spectrometry. Here, CSC was performed as previously described in detail (47-50), with

372 the exception that glycopeptide enrichment and bead washing was performed using an

373 epMotion 5073m (Eppendorf, Hamburg, Germany). For each sample, $750 \mu \mathrm{g}$ of total

374 peptide was diluted in binding buffer ( $80 \mathrm{mM}$ sodium phosphate, $2 \mathrm{M} \mathrm{NaCl}, 0.2 \%$ Tween 
$37520, \mathrm{pH} 7.8$ ) and incubated with $100 \mu \mathrm{L}$ of GenScript Streptavidin MagBeads (GenScript,

376 Piscataway, NJ) for $1 \mathrm{~h}$ with mixing. Beads were then washed sequentially with: (1) $2 \%$

377 sodium dodecyl sulfate in ultrapure water, (2) $80 \mathrm{mM}$ sodium phosphate, $2 \mathrm{M} \mathrm{NaCl}$,

$3780.2 \%$ Tween $20, \mathrm{pH} 7.8,(3) 100 \mathrm{mM}$ sodium carbonate, (4) $80 \%$ isopropyl alcohol in

379 ultrapure water, (5) ultrapure water, and (6) $50 \mathrm{mM}$ ammonium bicarbonate. Peptides

380 were released by digestion with PNGase F (Promega, Madison, WI) overnight at $37^{\circ} \mathrm{C}$

381 with vortexing. Deglycosylated peptides were cleaned and desalted using the SP2

382 procedure (73). Peptide Retention Time Calibration (PRTC) Mixture (ThermoFisher

383 Scientific) was added to each sample at a final concentration of $2 \mathrm{nM}$ to enable

384 retention time calibration and assessment of instrument performance throughout the

385 acquisition. A "pooled QC" mixture was generated by combining equal portions of each

386 sample. Individual samples were queued in a randomized order within a technical

387 injection series with two technical replicates each. Pooled QC samples were analyzed

388 at the beginning and end of each technical injection series. Samples were analyzed by

389 liquid chromatography tandem mass spectrometry (LC-MS/MS) using a Dionex UltiMate

3903000 RSLCnano system in-line with an Orbitrap Fusion Lumos (ThermoFisher

391 Scientific), and data were analysed with Proteome Discoverer 2.3 and Skyline (38).

392 Normalized quantitation ratios were determined by comparisons of each sample type to

393 the pooled QC.

394 Immunofluorescence microscopy

395 T cells were adhered onto poly-L-lysine-coated glass coverslips as described in (74)

396 and permeabilized in $0.5 \%$ saponin in PBS + 3\% BSA + $880 \mathrm{uM} \mathrm{Ca}^{2+}+490 \mathrm{uM} \mathrm{Mg}^{2+}$.

397 Permeabilized cells were incubated with primary antibodies, washed, and then 
398 incubated with secondary antibodies conjugated to a fluorophore. 4',6-diamidino-2-

399 phenylindole (DAPI) was added to the final PBS wash at $1 \mathrm{ug} / \mathrm{ml}$ to stain DNA.

400 Superresolution microscopy was performed on a Nikon Structured-Illumination

401 Microscope (N-SIM; Nikon) and NIS-Elements AR imaging and 3D reconstruction

402 software (v. 5.11) (Nikon Instruments Inc, Melville, NY). Images were taken using a

403 Nikon 100X Oil-immersion lens (CFI Apo SR TIRF 1.49 NA) and an Andor iXon+897

404 EMCCD camera. Confocal microscopy was performed on a Nikon Eclipse Ti2

405 microscope equipped with a W1 Spinning Disc, Orca Flash CMOS camera, and 100X

406 oil-immersion objective (CFI Plan Apo $\lambda 1.49$ NA), and NIS-Elements AR imaging and

$4073 \mathrm{D}$ reconstruction software (v. 6.0).

408 Flow cytometry

409 Cells were incubated with primary antibodies in $1 \%$ bovine serum albumin (BSA) in

410 DMEM -phenol red for 30 min on ice, washed, and incubated with secondary antibodies.

411 Flow cytometry was performed using an LSRII flow cytometer (BD Biosciences, San

412 Jose, CA). Data was analyzed using FlowJo analysis software (v. 10.7, BD

413 Biosciences). Infected cells were selectively analyzed by GFP+ gating. Non-viable cells

414 were excluded from all flow cytometric analyses.

\section{Immunoblotting}

416 Cell lysates were prepared using 1\% Triton Tx-100 lysis buffer supplemented with 62.5

$417 \mathrm{U} / \mathrm{ml}$ Benzonase and $174 \mu \mathrm{g} / \mathrm{ml}$ phenylmethylsulfonyl fluoride (PMSF) followed by the

418 addition of an equal volume of $2 \%$ sodium dodecyl sulfate (SDS) and $100 \mathrm{mM}$ Tris- $\mathrm{HCl}$

419 (pH 7.4). Lysates were normalized to total protein concentration as determined by 
420 bicinchronic acid (BCA) assay (Pierce, Rockford, IL). Lysates were resolved by SDS-

421 PAGE and transferred to BA-85 nitrocellulose membrane (Cytivia, Marlborough, MA).

422 Membranes were incubated with primary antibodies, followed by HRP-conjugated

423 secondary antibody (BioRad Laboratories, Hercules, CA). Bands were visualized using

424 SuperSignal West Pico reagent (ThermoFisher Scientific) imaged with an Azure c600

425 gel documentation system and quantified using AzureSpot software (v2.2.167) (Azure

426 Biosystems, Dublin, CA).

427 Inhibition of protein degradation

428 HHV6A-infected or uninfected JJhan cells were incubated in RPMI in $50 \mathrm{mM} \mathrm{NH}_{4} \mathrm{Cl}, 100$

429 nM MG-132, or a DMSO control for 19 hours. Cells were then divided for use in

430 immunofluorescence microscopy or immunoblot analysis.

$431 \quad q R T-P C R$

432 Cells were lysed in TRIzol (Invitrogen, Carlsbad, CA) according to the manufacturer's

433 instructions. Approximately 2 ug of RNA was treated with an AccuRT Genomic DNA

434 Removal Kit (Applied Biological Materials, Richmond, BC, Canada) prior to cDNA

435 synthesis using the 5X All-In-One Master mix (Applied Biological Materials), both

436 according to the manufacturer's instructions. qPCR was performed using a BioRad

437 CFX96 Real-Time System (BioRad Laboratories, Hercules, CA) and data analyzed

438 using BioRad CFX Maestro (v.4.1.2433.1219). CD45 RNA levels were normalized to a

439 cellular control for each sample (28S rRNA). Primer sets are listed in Table 1. All qPCR

440 reactions were run in technical triplicate with corresponding no template and -RT

441 controls, which did not exceed background levels. The delta-delta $\mathrm{C}_{\mathrm{t}}$ method was used 
442 to calculate the relative abundance of CD45 cDNA from HHV6A-infected JJhan cells

443 and 293T cells relative to the uninfected JJhan cell control.

444 Table 1. Primers used in this study.

\begin{tabular}{|l|l|}
\hline CD45 qRT4 FWD & AAAAGTGCTCCTCCAAGCCA \\
\hline CD45 qRT4 REV & TGGGAGGCCTACACTTGACA \\
\hline 28S rRNA 3783F & GTGACGCGCATGAATGGA \\
\hline 28S rRNA 3846R & TGTGGTTTCGCTGGATAGTAGGT \\
\hline
\end{tabular}

445 Statistical analysis

446 Statistical analysis was done using GraphPad 8 (v.8.4.3). One-way ANOVA analysis

447 with Tukey's multiple comparisons test was used to compare the abundance of CD45

448 mRNA 293T cells, uninfected JJhan cells, and HHV6A-infected T cells. ${ }^{* * *}<0.0001$

\section{Acknowledgements}

450 The authors thank Rebekah Mokry for helpful discussion, Drs. Benedikt Kaufer, Arthur

451 Weiss, Vera Tarakanova, Ken Brockman, and Scott Terhune for generous provision of

452 reagents and helpful discussion. We acknowledge funding from National Institutes of

453 Health under Award Numbers AI123745 to AWH and RLG, HL134010 to RLG, and the

454 American Heart Association 20PRE35200049 to LBL. LBL is a member of the MCW

455 Medical Scientist Training Program, partially supported by NIH T32 GM080202. 


\section{References Cited}

1. Zerr DM, Meier AS, Selke SS, Frenkel LM, Huang ML, Wald A, Rhoads MP, Nguy L, Bornemann R, Morrow RA, Corey L. 2005. A population-based study of primary human herpesvirus 6 infection. N Engl J Med 352:768-76.

2. Yamanishi K, Shiraki K, Kondo T, Okuno T, Takahashi M, Asano Y, Kurata T. 1988. Identification of human herpesvirus- 6 as a causal agent for exanthem subitum The Lancet 331:1065-1067.

3. Omasits U, Ahrens $\mathrm{CH}$, Müller S, Wollscheid B. 2014. Protter: interactive protein feature visualization and integration with experimental proteomic data. Bioinformatics 30:884-6.

4. Emmanual J. Wiertz TRJ, Lei Sun, Matthew Bogyo, Hans J. Geuze, Hidde L. Ploegh. 1996. The Human Cytomegalovirus US11 Gene ProductDislocates MHC Class I Heavy Chainsfrom the Endoplasmic Reticulum to the Cytosol. Cell 84:769-779.

5. Jones TR, Sun L. 1997. Human cytomegalovirus US2 destabilizes major histocompatibility complex class I heavy chains. J Virol 71:2970-2979.

6. Elboim M, Grodzovski I, Djian E, Wolf DG, Mandelboim O. 2013. HSV-2 specifically down regulates HLA-C expression to render HSV-2-infected DCs susceptible to NK cell killing. PLoS Pathog 9:e1003226.

7. Imai T, Koyanagi N, Ogawa R, Shindo K, Suenaga T, Sato A, Arii J, Kato A, Kiyono H, Arase H, Kawaguchi Y. 2013. Us3 kinase encoded by herpes simplex virus 1 mediates downregulation of cell surface major histocompatibility complex class I and evasion of CD8+ T cells. PLoS One 8:e72050.

8. D.J. Schust ABH, H. L. Ploegh. 1996. Herpes simplex virus blocks intracellular transport of HLA-G in placentally derived human cells. Journal of Immunology 157:3375-3380.

9. Früh K, Ahn K, Djaballah H, Sempe P, van Endert P, Tampe R, Peterson P, Yang Y. 1995. A viral inhibitor of peptide transporters for antigen presentation. Nature 375:415-418.

10. A.York CR, D. W. Andrews, S. R. Riddell, F. L. Graham, D. C. Johnson. 1994. A cytosolic herpes simplex virus protein inhibits antigen presentation to CD8+ T lymphocytes Cell 20:525-535.

11. Keating S, Prince S, Jones M, Rowe M. 2002. The lytic cycle of Epstein-Barr virus is associated with decreased expression of cell surface major histocompatibility complex class I and class II molecules. J Virol 76:8179-88.

12. Glosson NL, Hudson A. 2007. Human herpesvirus-6A and $-6 B$ encode viral immunoevasins that downregulate class I MHC molecules. Virology 365:125-135.

13. Hudson AW, Howley PM, Ploegh HL. 2001. A human herpesvirus 7 glycoprotein, U21, diverts major histocompatibility complex class I molecules to lysosomes. J Virol 75:12347-58.

14. Dassa L, Seidel E, Oiknine-Djian E, Yamin R, Wolf DG, Le-Trilling VTK, Mandelboim O. 2018. The Human Cytomegalovirus Protein UL148A Downregulates the NK Cell-Activating Ligand MICA To Avoid NK Cell Attack. J Virol 92.

15. Seidel E, Le VTK, Bar-On Y, Tsukerman P, Enk J, Yamin R, Stein N, Schmiedel D, Oiknine Djian E, Weisblum $Y$, Tirosh B, Stastny P, Wolf DG, Hengel H, Mandelboim O. 2015. Dynamic Co-evolution of Host and Pathogen: HCMV Downregulates the Prevalent Allele MICA*008 to Escape Elimination by NK Cells. Cell Rep 10:968-982. 
16. Fielding CA, Aicheler R, Stanton RJ, Wang EC, Han S, Seirafian S, Davies J, McSharry BP, Weekes MP, Antrobus PR, Prod'homme V, Blanchet FP, Sugrue D, Cuff S, Roberts D, Davison AJ, Lehner PJ, Wilkinson GW, Tomasec P. 2014. Two novel human cytomegalovirus NK cell evasion functions target MICA for lysosomal degradation. PLoS Pathog 10:e1004058.

17. Stern-Ginossar N, Elefant N, Zimmermann A, Wolf DG, Saleh N, Biton M, Horwitz E, Prokocimer Z, Prichard M, Hahn G, Goldman-Wohl D, Greenfield C, Yagel S, Hengel H, Altuvia Y, Margalit H, Mandelboim O. 2007.

18. Rölle A, Mousavi-Jazi M, Eriksson M, Odeberg J, Söderberg-Nauclér C, Cosman D, Kärre K, Cerboni C. 2003. Effects of human cytomegalovirus infection on ligands for the activating NKG2D receptor of NK cells: upregulation of UL16-binding protein (ULBP)1 and ULBP2 is counteracted by the viral UL16 protein. The Journal of Immunology 171:902-908.

19. Schneider CL, Hudson A. 2011. The human herpesvirus-7 (HHV-7) U21 immunoevasin subverts NKmediated cytoxicity through modulation of MICA and MICB. PLoS Pathog 7:e1002362.

20. Anders S, Huber W. 2010. Differential expression analysis for sequence count data. Genome Biol 11:R106.

21. Bonifacino JS, Dell'Angelica EC. 2001. Immunoprecipitation. Current protocols in cell biology / editorial board, Juan S Bonifacino [et al] Chapter 7:Unit 7.2.

22. Peretti S, Shaw A, Blanchard J, Bohm R, Morrow G, Lifson JD, Gettie A, Pope M. 2005. Immunomodulatory effects of HSV-2 infection on immature macaque dendritic cells modify innate and adaptive responses. Blood 106:1305-13.

23. Morrow G, Slobedman B, Cunningham AL, Abendroth A. 2003. Varicella-zoster virus productively infects mature dendritic cells and alters their immune function. J Virol 77:4950-9.

24. Mikloska Z, Bosnjak L, Cunningham AL. 2001. Immature monocyte-derived dendritic cells are productively infected with herpes simplex virus type 1. J Virol 75:5958-64.

25. Andrews DM, Andoniou CE, Granucci F, Ricciardi-Castagnoli P, Degli-Esposti MA. 2001. Infection of dendritic cells by murine cytomegalovirus induces functional paralysis. Nat Immunol 2:1077-84.

26. Salio M, Cella M, Suter M, Lanzavecchia A. 1999. Inhibition of dendritic cell maturation by herpes simplex virus. Eur J Immunol 29:3245-53.

27. Jones D, Como CN, Jing L, Blackmon A, Neff CP, Krueger O, Bubak AN, Palmer BE, Koelle DM, Nagel MA. 2019. Varicella zoster virus productively infects human peripheral blood mononuclear cells to modulate expression of immunoinhibitory proteins and blocking PD-L1 enhances virus-specific CD8+ T cell effector function. PLoS Pathog 15:e1007650.

28. Gilardini Montani MS, Santarelli R, Falcinelli L, Gonnella R, Granato M, Di Renzo L, Cuomo L, Vitillo M, Faggioni A, Cirone M. 2018. EBV up-regulates PD-L1 on the surface of primary monocytes by increasing ROS and activating TLR signaling and STAT3. J Leukoc Biol 104:821-832.

29. Host KM, Jacobs SR, West JA, Zhang Z, Costantini LM, Stopford CM, Dittmer DP, Damania B. 2017. Kaposi's Sarcoma-Associated Herpesvirus Increases PD-L1 and Proinflammatory Cytokine Expression in Human Monocytes. mBio 8. 
30. McSharry BP, Forbes SK, Cao JZ, Avdic S, Machala EA, Gottlieb DJ, Abendroth A, Slobedman B. 2014. Human cytomegalovirus upregulates expression of the lectin galectin 9 via induction of beta interferon. J Virol 88:10990-4.

31. Reddy PB, Sehrawat S, Suryawanshi A, Rajasagi NK, Mulik S, Hirashima M, Rouse BT. 2011. Influence of galectin-9/Tim-3 interaction on herpes simplex virus-1 latency. J Immunol 187:5745-55.

32. Benedict CA, Loewendorf A, Garcia Z, Blazar BR, Janssen EM. 2008. Dendritic cell programming by cytomegalovirus stunts naive T cell responses via the PD-L1/PD-1 pathway. J Immunol 180:4836-47.

33. Weekes MP, Tomasec P, Huttlin EL, Fielding CA, Nusinow D, Stanton RJ, Wang ECY, Aicheler R, Murrell I, Wilkinson GWG, Lehner PJ, Gygi SP. 2014. Quantitative temporal viromics: an approach to investigate host-pathogen interaction. Cell 157:1460-1472.

34. Bartee E, McCormack A, Früh K. 2006. Quantitative membrane proteomics reveals new cellular targets of viral immune modulators. PLoS Pathog 2:e107.

35. Hertel L, Lacaille VG, Strobl H, Mellins ED, Mocarski ES. 2003. Susceptibility of immature and mature Langerhans cell-type dendritic cells to infection and immunomodulation by human cytomegalovirus. J Virol 77:7563-74.

36. Coscoy L, Ganem D. 2001. A viral protein that selectively downregulates ICAM-1 and B7-2 and modulates T cell costimulation. J Clin Invest 107:1599-606.

37. Mansouri M, Douglas J, Rose PP, Gouveia K, Thomas G, Means RE, Moses AV, Früh K. 2006. Kaposi sarcoma herpesvirus K5 removes CD31/PECAM from endothelial cells. Blood 108:1932-40.

38. MacLean B, Tomazela DM, Shulman N, Chambers M, Finney GL, Frewen B, Kern R, Tabb DL, Liebler DC, MacCoss MJ. 2010. Skyline: an open source document editor for creating and analyzing targeted proteomics experiments. Bioinformatics 26:966-8.

39. Rheinlander A, Schraven B, Bommhardt U. 2018. CD45 in human physiology and clinical medicine. Immunol Lett 196:22-32.

40. Holmes N. 2006. CD45: all is not yet crystal clear. Immunology 117:145-55.

41. Hermiston ML, Xu Z, Weiss A. 2003. CD45: a critical regulator of signaling thresholds in immune cells. Annu Rev Immunol 21:107-37.

42. Sasaki T, Sasaki-Irie J, Penninger JM. 2001. New insights into the transmembrane protein tyrosine phosphatase CD45. Int J Biochem Cell Biol 33:1041-6.

43. Koretzky GA, Picus J, Schultz T, Weiss A. 1991. Tyrosine phosphatase CD45 is required for T-cell antigen receptor and CD2-mediated activation of a protein tyrosine kinase and interleukin 2 production. Proc Natl Acad Sci U S A 88:2037-41.

44. Koretzky GA, Picus J, Thomas ML, Weiss A. 1990. Tyrosine phosphatase CD45 is essential for coupling Tcell antigen receptor to the phosphatidyl inositol pathway. Nature 346:66-8.

45. Pingel JT, Thomas ML. 1989. Evidence that the leukocyte-common antigen is required for antigen-induced T lymphocyte proliferation. Cell 58:1055-65. 
46. Fujinaka CM, Waas M, Gundry RL. 2018. Mass Spectrometry-Based Identification of Extracellular Domains of Cell Surface N-Glycoproteins: Defining the Accessible Surfaceome for Immunophenotyping Stem Cells and Their Derivatives. Methods Mol Biol 1722:57-78.

47. Mallanna SK, Waas M, Duncan SA, Gundry RL. 2017. N-glycoprotein surfaceome of human induced pluripotent stem cell derived hepatic endoderm. Proteomics 17.

48. Mallanna SK, Cayo MA, Twaroski K, Gundry RL, Duncan SA. 2016. Mapping the Cell-Surface NGlycoproteome of Human Hepatocytes Reveals Markers for Selecting a Homogeneous Population of iPSCDerived Hepatocytes. Stem Cell Reports 7:543-556.

49. Boheler KR, Bhattacharya S, Kropp EM, Chuppa S, Riordon DR, Bausch-Fluck D, Burridge PW, Wu JC, Wersto RP, Chan GC, Rao S, Wollscheid B, Gundry RL. 2014. A human pluripotent stem cell surface Nglycoproteome resource reveals markers, extracellular epitopes, and drug targets. Stem Cell Reports 3:185-203.

50. Wollscheid B, Bausch-Fluck D, Henderson C, O'Brien R, Bibel M, Schiess R, Aebersold R, Watts JD. 2009. Mass-spectrometric identification and relative quantification of $\mathrm{N}$-linked cell surface glycoproteins. Nat Biotechnol 27:378-86.

51. Pawelec G, Borowitz A, Krammer PH, Wernet P. 1982. Constitutive interleukin 2 production by the JURKAT human leukemic T cell line. Eur J Immunol 12:387-392.

52. Dölken L, Krmpotić A, Kothe S, Tuddenham L, Tanguy M, Marcinowski L, Ruzsics Z, Elefant N, Altuvia Y, Margalit H, Koszinowski UH, Jonjić S, Pfeffer S. 2010. Cytomegalovirus microRNAs facilitate persistent virus infection in salivary glands. PLoS Pathog 6:e1001150.

53. Hall LR, Streuli M, Schlossman SF, Saito H. 1988. Complete exon-intron organization of the human leukocyte common antigen (CD45) gene. J Immunol 141:2781-7.

54. Streuli M, Hall LR, Saga Y, Schlossman SF, Saito H. 1987. Differential usage of three exons generates at least five different mRNAs encoding human leukocyte common antigens. J Exp Med 166:1548-66.

55. Ralph SJ, Thomas ML, Morton CC, Trowbridge IS. 1987. Structural variants of human T200 glycoprotein (leukocyte-common antigen). Embo j 6:1251-7.

56. Rothstein DM, Saito H, Streuli M, Schlossman SF, Morimoto C. 1992. The alternative splicing of the CD45 tyrosine phosphatase is controlled by negative regulatory trans-acting splicing factors. J Biol Chem 267:7139-47.

57. Sullivan BM, Coscoy L. 2008. Downregulation of the T-cell receptor complex and impairment of T-cell activation by human herpesvirus 6 u24 protein. J Virol 82:602-8.

58. Nicholas J. 1996. Determination and analysis of the complete nucleotide sequence of human herpesvirus7. J Virol 70:5975-5989.

59. Isegawa Y, Mukai T, Nakano K, Kagawa M, Chen J, Mori Y, Sunagawa T, Kawanishi K, Sashihara J, Hata A, Zou P, Kosuge H, Yamanishi K. 1999. Comparison of the complete DNA sequences of human herpesvirus 6 variants A and B. J Virol 73:8053-63.

60. Thiel N, Keyser KA, Lemmermann NA, Oduro JD, Wagner K, Elsner C, Halenius A, Lenac Rovis T, Brinkmann MM, Jonjic S, Cicin-Sain L, Messerle M. 2016. The Mouse Cytomegalovirus Gene m42 Targets Surface 
Expression of the Protein Tyrosine Phosphatase CD45 in Infected Macrophages. PLoS Pathog 12:e1006057.

61. Mustelin T, Coggeshall KM, Altman A. 1989. Rapid activation of the T-cell tyrosine protein kinase pp56lck by the CD45 phosphotyrosine phosphatase. Proc Natl Acad Sci U S A 86:6302-6.

62. Mustelin T, Altman A. 1990. Dephosphorylation and activation of the T cell tyrosine kinase pp56lck by the leukocyte common antigen (CD45). Oncogene 5:809-13.

63. Mustelin T, Pessa-Morikawa T, Autero M, Gassmann M, Andersson LC, Gahmberg CG, Burn P. 1992. Regulation of the p59fyn protein tyrosine kinase by the CD45 phosphotyrosine phosphatase. Eur J Immunol 22:1173-8.

64. Gabaev I, Steinbruck L, Pokoyski C, Pich A, Stanton RJ, Schwinzer R, Schulz TF, Jacobs R, Messerle M, KayFedorov PC. 2011. The human cytomegalovirus UL11 protein interacts with the receptor tyrosine phosphatase CD45, resulting in functional paralysis of T cells. PLoS Pathog 7:e1002432.

65. Zischke J, Mamareli P, Pokoyski C, Gabaev I, Buyny S, Jacobs R, Falk CS, Lochner M, Sparwasser T, Schulz TF, Kay-Fedorov PC. 2017. The human cytomegalovirus glycoprotein pUL11 acts via CD45 to induce T cell IL-10 secretion. PLoS Pathog 13:e1006454.

66. Arena A, Liberto MC, lannello D, Capozza AB, Focà A. 1999. Altered cytokine production after human herpes virus type 6 infection. New Microbiol 22:293-300.

67. Li C, Goodrich JM, Yang X. 1997. Interferon-gamma (IFN-gamma) regulates production of IL-10 and IL-12 in human herpesvirus-6 (HHV-6)-infected monocyte/macrophage lineage. Clin Exp Immunol 109:421-5.

68. Windheim M, Southcombe JH, Kremmer E, Chaplin L, Urlaub D, Falk CS, Claus M, Mihm J, Braithwaite M, Dennehy K, Renz H, Sester M, WatzI C, Burgert HG. 2013. A unique secreted adenovirus E3 protein binds to the leukocyte common antigen CD45 and modulates leukocyte functions. Proc Natl Acad Sci U S A 110:E4884-93.

69. Sanyal A, Wallaschek N, Glass M, Flamand L, Wight DJ, Kaufer BB. 2018. The ND10 Complex Represses Lytic Human Herpesvirus 6A Replication and Promotes Silencing of the Viral Genome. Viruses 10.

70. Courtney AH, Amacher JF, Kadlecek TA, Mollenauer MN, Au-Yeung BB, Kuriyan J, Weiss A. 2017. A Phosphosite within the SH2 Domain of Lck Regulates Its Activation by CD45. Mol Cell 67:498-511.e6.

71. Tang H, Kawabata A, Yoshida M, Oyaizu H, Maeki T, Yamanishi K, Mori Y. 2010. Human herpesvirus 6 encoded glycoprotein Q1 gene is essential for virus growth. Virology 407:360-367.

72. May NA, Wang Q, Balbo A, Konrad SL, Buchli R, Hildebrand WH, Schuck P, Hudson AW. 2014. Human herpesvirus 7 U21 tetramerizes to associate with class I major histocompatibility complex molecules. J Virol 88:3298-308.

73. Waas M, Pereckas M, Jones Lipinski RA, Ashwood C, Gundry RL. 2019. SP2: Rapid and Automatable Contaminant Removal from Peptide Samples for Proteomic Analyses. J Proteome Res 18:1644-1656.

74. Mihara K, Nakayama T, Saitoh H. 2015. A Convenient Technique to Fix Suspension Cells on a Coverslip for Microscopy. Curr Protoc Cell Biol 68:4 30 1-4 3010. 